\title{
XIII. Silicomagnesiofluorit, ein neues Mineral von Luppiko in Finnland.
}

\author{
Von
}

P. Zemiattschenski in St. Petersburg.

Das in Rede stehende Mineral ist in den Bergwerksabfällen des Ortes Luppiko in der Umgegend des bekannten Pitküranta in Finnlind in Gestalt eines Blockes von einigen Kilogramm Gewicht gefunden worden. Es bildet radialstrahlige Aggregate von meist halbkugeliger, seltener von Kugelgestalt. Die Länge der Fasern schwankt zwischen einigen Millimetern und $1-1,5 \mathrm{~cm}$. Allem Anscheine nach befindet es sich auf Serpentin. Wenigstens hat sich hier und da milchweißer compacter Serpentin mit trüben Quarzkörnern erhalten. Unmittelbar an diesen schließt sich eine Zone eines feinnadeligen Minerals an, worin die Bestandteile unregelmäßig angeordnet sind oder die radialstrahlige Anordnung undeutlich hervortritt. jie Breite dieser Zone beträgt etwa $1 \mathrm{~cm}$.

Auf die feinnadelige Zone folgt eine solche von größeren, halbkugelförmigen, radialstrahligen Mineralausscheidungen, wobei sich eine gewisse Stufenfolge im Übergange von feineren Nadeln $\mathrm{zu}$ gröberen beobachten läßt.

Die Farbe des Minerals ist aschgrau oder licht grünlich und bläulich mit seidenartigem Glanz. Die feinnadelige Zone zeigt stellenweise eine zrelbliche Fürbung und zuweilen ist ein Teil eines nadelförmigen Individuums gelblich, der andere aschgrau gefärbt.

Die Härte ist $\mathbf{2 , 5}$.

Das von Herrn v. Isküll mit Hilfe des Pyknometers bestimmte spec. Gewicht beträgt 2,9125 bei $20^{\circ} \mathrm{C}$.

Die Doppelbrechung ist schwach und positiv, die Auslöschungsschiefe ist parallel der Längsrichtung der Fasern.

In Gestalt von Nadeln schmilzt das Mineral leicht vor dem Lötrohre und bildet ein trübes grünliches Glas. Grüßere Stücke lassen sich nur an den Kanten schwach anschmelzen. Ebenso erfolgt die Schmelzung auch in der Flamme des Bunsenbrenners. 
Die Bestimmung des Schmelzpunktes mittels Le Chatelier-Pyrometer ergab 9620. Einen zweiten (kurzen) Anhaltspunkt bemerkt man beim Steigen der Temperatur bei $874^{\circ}$ (Mittel).

In einem Glasröhrchen erhitzt scheidet das Mineral ein bemerkbares Quantum Wasser von alkalischer Reaction aus. Ebenso reagieren auch die aus dem offenen Ende des Rohres entweichenden Dämpfe. Die dabei ausströmenden Gase haben einen deutlichen Geruch nach verbrannten Federn, was auf die Anwesenheit einer gewissen Menge organischer Substanz im Mineral hinweist. Durch diesen Umstand läßt sich ein geringes Dunkelwerden des Minerals zu Beginn des Glühprozesses erklären.

Die Wände des Röhrchens zeigen hier und da trübe Flecken, woraus man auf das Vorhandensein von Fluorwasserstoff in den flüchtigen Producten schließen kann. Mit saurem schwefelsaurem Kali gibt das Mineral eine starke Reaction auf Fluor. Das mit Wasser angefeuchtete Mineralpulver gibt auf Lackmuspapier deutlich alkalische Reaction, die nach dem Durchglühen im Glasröhrchen noch stärker wird.

In Salz-, Salpeter- und Schwefelsäure löst sich das Mineral leicht ohne Rest auf. Bei Behandlung mit Schwefelsäure braust das Pulver stürmisch auf.

Wie die vorläufige qualitative $\Lambda$ nalyse ergeben hat, sind die wesentlichen Bestandteile des Minerals $\mathrm{SiO}_{2}, \mathrm{MgO}, \mathrm{H}_{2} \mathrm{O}$ und $\mathrm{F}$. Außer diesen ist in geringer Menge $\mathrm{H} C \mathrm{O}, \mathrm{MnO}, \mathrm{SO}_{3}$ und organische Substanz vorhanden. Bekanntlich ist die quantitative Bestimmung bei gleichzeitigem Vorhandensein von $\mathrm{SiO}_{2}, \mathrm{H}_{2} \mathrm{O}$ und $\mathrm{F}^{\prime}$ mit beträchtlichen Schwierigkeiten verknüpft. Für das in Rede stehende Mineral erwies sich als das beste Verfahren zur Beslimmung des Fluors die Analyse mit Schwefelsäure in Gegenwarl von geglühtem Quarzpulver ${ }^{1}$ ), während alle übrigen unbefriedigende Resultate lieferten.

Die für Fluor enthaltende Silicate empfohlene Bestimmung des Wassers (namentlich das Ausglühen mit Bleioxyd) hatte bei dem besprochenen Mineral dank der leichten Bildung und Verflüchtigung von Fluorwasserstoff keine guten Ergebnisse. Deshalb ist der Wassergehalt auf indirectem Wege ermiltelt worden. Zieht man nämlich in Betracht, daß das Mineral bei anhaltendem Glühen sein Fluor allmählich in Sauerstoff umsetzen muß und kennt man die Menge des letzteren, so kann man berechnen, wie groß der Verlust bei vollem Ersatze des Fluors durch Sauerstoff sein muß. Der factische Verlust bei andauerndem Glühen (im Verlaufe von 20 Stunden) bis zum constanten Gewicht ergab $21,437 \%$, während der berechnete für den Ersatz des Fluors $(31,011 \%)$ durch Sauerstoff sich auf 18,420\% stellte. Demnach beträgt die Differenz 3,017 und diese Ziffer muß den Wassergehalt bezeichnen. Hieraus ist die Menge des Wasserstoffes berechnet

1) R. Fresenius, Anl. z. (puant. chem. Anal. 1875, 1, 431. 
worden. Es ist indes zu beachten, daß eigentlich etwas weniger Wasser vorhanden sein müßte, da beim Ausglühen auch die organische Substanz eliminiert wird, allein bei ihrer geringen Menge kann sie ignoriert werden.

Das Ergebnis der Analyse des Minerals lüßt sich folgendermaßen darstellen (manche von den Zahlen sind der Mittelwert von fünf Analysen, andere von zweien):

$\begin{array}{lr} & \text { Mittel: } \\ \mathrm{SiO}_{2} & 19,857 \\ \mathrm{CaO} & 38,476 \\ \mathrm{MgO} & \mathbf{1 8 , 2 7 2} \\ \mathrm{H}_{2} \mathrm{O}_{3} & 2,303 \\ \mathrm{Nn}_{3} \mathrm{O}_{4} & 0,062 \\ \mathrm{SO}_{3} & 0,273 \\ \mathrm{H}_{2} \mathrm{O} & 5,895 \\ \mathrm{~F} & 31,011 \\ \mathrm{Si} & 9,337 \\ \mathrm{Ca} & 27,538 \\ \mathrm{MIg} & 11,028 \\ \mathrm{He} & 1,611 \\ \mathrm{Mn} & 0,017 \\ \mathrm{II} & 0,335 \\ \mathrm{H} & 31,011 \\ \mathrm{SO}_{3} & 0,273 \\ \mathrm{O} \text { (aus dem leste) } & 18,850\end{array}$

Auf Grund der mikroskopischen Untersuchung kann man einen Teil des Eisens in Gestalt von Oxyd als mechanische Beimengung betrachten (ebenso das geringe Quantum an Mangan). Der Rest des Eisens ist aber als Oxydul vorhanden und es ist sehr wahrscheinlich, daß er isomorph die Magnesia im Silicat ersetzt. Lassen wir auch das Mangan, das Schwefelsäureanhydrid und das minimale Quantum an organischer Substanz unberücksichtigt, so erhalten wir für die Hauptbestandteile des Minerals folgende Atomverhältnisse :

Atomverhältnis :

\begin{tabular}{|c|c|c|c|}
\hline$S i$ & 0,330 & 1 & 2 \\
\hline $\mathrm{Ca}$ & 0,687 & 2,08 & 4 \\
\hline$M g$ & $0,452\}_{0,481}$ & 1,43 & \\
\hline$F_{c}$ & $0,029 \int 0,401$ & & \\
\hline$H$ & 0,335 & 1,01 & 2 \\
\hline$F$ & 1,632 & 4,94 & 10 \\
\hline$O$ & 1,180 & 3,57 & 7 \\
\hline
\end{tabular}

Somit kann die allgemeine chemische Formel für das besprochene Mineral wie folgt aufgestellt werden: $\mathrm{H}_{2} \mathrm{Ca}_{4} \mathrm{Mg}_{3} \mathrm{Si}_{2} \mathrm{O}_{7} \mathrm{~F}_{10}$. Berechnen wir 
hieraus das Procentverhältnis und vergleichen wir es mit den durch die Analyse ermittelten Werten, so erhalten wir:

$\begin{array}{lccc} & \text { Gefunden: } & \text { Berechnet: } & \text { Dilferenz: } \\ S i & 9,337 & 9,81 & +0,47 \\ \mathrm{Ca} & 27,538 & 27,00 & -0,54 \\ \mathrm{Mg} & 11,028 & 12,149 & +1,12 \\ \mathrm{Fe} & \mathbf{1 , 6 1 1} & - & - \\ H & 0,335 & 0,352 & +0,017 \\ F & 31,011 & 32,06 & +1,05 \\ O & \mathbf{1 8 , 8 5 0} & 18,89 & +0,04\end{array}$

Im allgemeinen entspricht das aus der theoretischen Formel berechnete Procentverhältnis der Bestandteile recht befriedigend dem bei der Analyse gefundenen. Die größte $\Lambda$ bweichung zeigt sich beim Magnesium und beim Fluor, was ganz begreiflich ist, denn der procentuelle Gehalt ist nur für das Magnesium berechnet worden, während ein Teil davon durch Eisen ersetzt worden ist. Inbetreff des Fluors ist $\mathrm{zu}$ bemerken, daß ein Teil davon durch Schwefelsäureanhydrid ersetzt sein kann, dessen Anwesenheit die Analyse dargetan hat.

Viel schwieriger ist es, dem Mineral eine einigermaßen begründete Constitutionsformel zu geben. Vereinigen wir $C a$ und $M g$ unter der Bezeichnung $\stackrel{\mathrm{II}}{R}$, so erhalten wir den einfachsten Ausdruck:

$$
2\left(\mathrm{II}_{\mathrm{SiO}}\right) .5 \stackrel{\mathrm{II}}{\mathrm{R}} \mathrm{F}_{2} . \mathrm{H}_{2} \mathrm{O} \text {, }
$$

wobei $\mathrm{Ca}: M g=4: 3$ ist. Allein wir haben Grund anzunehmen, daß der Wasserstoff in Gestalt einer Hydroxylgruppe und nicht als Krystallisationswasser vorhanden ist, denn er verflüchtigt sich nicht leicht beim Ausglühen: bei der Erhitzung bis auf $300^{\circ}-340^{\circ} \mathrm{C}$. macht sich nur eine ganz geringe Vergrößlerung des Verlustes im Vergleich mit dem bei $100^{\circ} \mathrm{C}$. bemerkbar. Bei der Erhitzung bis auf $100^{\circ} \mathrm{C}$. verliert das Mineral 0,401\%, bei der Erhitzung auf $300^{0}-340^{\circ} \mathrm{C}$. beläuft sich der Verlust auf 0,783\% und hierher gehören gewiß auch die organischen Substanzen. Außerdem hat es sich herausgestellt, daß bei Erhitzung des Minerals auf eine Temperatur, die sich $600^{\circ} \mathrm{C}$. nähert, das Wasser und ein Teil des Fluors ziemlich leicht entfernt wird. Überhaupt verschwindet ein gewisser Teil des Fluors beim Erhitzen und wird leichter durch Sauerstoff ersetzt, als der andere. Bei Rotglut betrug nämlich der Gewichtsverlust des Minerals im Verlaufe von 1:. Stunden 6,498\%. Die weitere Erhitzung ergab im Laufe derselben $1 \frac{1}{2}$ Stunden $1,051 \%$ Verlust. Ein eben solcher Verlust erfolgte im Laufe von 12 Stunden und fiel beim weiteren Glühen während $1 \frac{1}{2}$ Stunden auf $0,043 \%$. Bei fortgesetzter Erhitzung fast bis zur Weißglut wächst der 
Verlust an Fluor und geht ohne Veränderung bis zu dessen völligem Verschwinden fort.

Abgesehen davon ist das Verhalten des Minerals in gepulvertem $\mathrm{Zu}$ stande bei schneller Behandlung (im Laufe weniger Minuten) mit Salpetersüure zu berücksichtigen. Dabei gehen $C a$ und $M g$ in anderem Verhältnisse in die Lösung über, als sie im Gesamtgehalte vorhanden sind: im ersten Falle $C a: M g=2: 1$, im zweiten, wie wir oben gesehen haben, $C a: M g$ $=4: 3$.

Auf Grund der vorgeführten Umstände scheint es mir zulässig, folgende chemische Formel aufzustellen, in der wenigstens zum T'eil seine Eigentümlichkeiten zum Ausdruck kommen:

$$
\mathrm{Mg}(\mathrm{HO}) \mathrm{F} . \mathrm{MgSiO}_{3} \cdot \mathrm{Ca}(\mathrm{HO}) \mathrm{F} \cdot \mathrm{CaSiO}_{2} \mathrm{~F}_{2} \cdot \mathrm{CCaF}_{2}, \mathrm{MgF}_{2} \text {. }
$$

Was die Benennung des Minerals betrifft, so gehe ich solchen, die mit dem Fundorte, mit der Ehrung dieser oder jener Person oder anderen zufälligen Umstïnden zusammenhängen, aus dem Wege und halte es für das Zweckmüßigste, das Mineral auf Grund seiner eigentümlichen chemischen Zusammensetzung als Silicomagnesiofluorit zu bezeichnen. 